\title{
Effect of Visual Acuity on Ocular Deviation
}

\section{Partha Haradhan Chowdhury ${ }^{1 *}$ and Brinda Haren Shah ${ }^{2}$}

${ }^{1}$ M. Optom, Department of Optometry, Shree Satchandi Jankalyan Samiti Netra Prasikshan Sansthan, Pauri, Affiliated to Uttarakhand State Medical Faculty, Dehradun, India

Research Article

Volume 3 Special Issue 1

Received Date: July 30, 2018

Published Date: August 15, 2018

${ }^{2}$ M. Optom, Department of Optometry, Gujarat University, Gujarat, India

*Corresponding author: Partha Haradhan Chowdhury, M. Optom, Department of Optometry, Shree Satchandi Jankalyan Samiti Netra Prasikshan Sansthan, Pauri, Affiliated to Uttarakhand State Medical Faculty, Dehradun, India, Email: optometrypublish@gmail.com

\section{Abstract}

Purpose: The aim of the present study is to correlate effect of visual acuity in the presence of ocular deviation.

Methods: A pilot, cross sectional, observational study was performed at tertiary eye care centers. Subjects with Ocular deviation between 10 to 40 prism diopters, Corrected distance Visual Acuity should be greater than 6/18 and Age should be between 10 to 40 years of age were included in the study. Visual acuity was assessed with Log Mar chart and recorded in log units.

Results: 30 subjects were included in the study. Out of that 16 subjects were in the age group of 11-20 years, 12 subjects were in the age group of 21-30 years and 2 subjects were in the age group of 31-40 years. $60 \%$ subjects were Female and $40 \%$ subjects were Male. Mean scores of visual parameters were taken. Visual acuity was deteriorated to 0.193 log units

Conclusions: In cases of ocular deviation, visual acuity deteriorates significantly.

Keywords: Ocular Deviation; Visual Acuity

\section{Introduction}

To maintain the binocular single vision, ocular deviation plays an important role. Because, in case of normal deviation, images are falling on the fovea and maintain binocular single vision. But it is not mandatory, that to maintain Binocular Single Vision, images should be focused on the fovea. If image of one eye is placed at the nasal fovea and image of other eye is focused on temporal fovea, then also there are chances to maintain Binocular
Vision. In most of the cases, Binocular Single Vision is not maintained in cases of Ocular Deviation [1]. In the presence of Ocular Deviation, an image of an object is placed at the para foveal region according to the deviation [2]. In cases of Eso deviation, images are focused at nasal Para foveal region and in case of Exo deviation; images are focused at Temporal Parafoveal region [3]. In these cases, there may be chances to deterioratation of visual 


\section{Open Access Journal of Ophthalmology}

acuity due to cone cell variation in the macular region. In the fovea, number of cone cells is highest compare to other region [4].

\section{Methodology}

A pilot, cross sectional, observational study was performed at tertiary eye care centers. Subjects with Ocular deviation between 10 to 40 prism diopters, Corrected distance Visual Acuity should be greater than 6/18, Age should be between 10 to 40 years of age and Ocular Deviation should be between 10 to 40 Prism Diopters were included in the study. Individuals with any other systemic disease(specially which can affect study), Individuals with any other Ocular Pathology, with any active ocular infection, any ocular anomalies like Corneal Scar etc ,ocular deviation if less than 10 degree and Significant amount of amblyopic patient were excluded from the study. Full refractive correction along with detailed fundus evaluation was performed in each and every patient. Visual acuity was assessed with Log Mar chart and recorded in log units. All the patients were included after oral/written consent.

\section{Results}

30 subjects were included in the study. Figure 1 shows distribution of subjects in various age groups.16 subjects were in the age group of 11-20 years, 12 subjects were in the age group of 21-30 years and 2 subjects were in the age group of 31-40 years. Figure 2 shows gender wise distribution of the subjects. $60 \%$ subjects were Female and $40 \%$ subjects were Male. Mean scores of visual parameters were taken using SPSS Software version 20. Figure 3 shows Visual acuity was deteriorated to 0.193 $\log$ units.

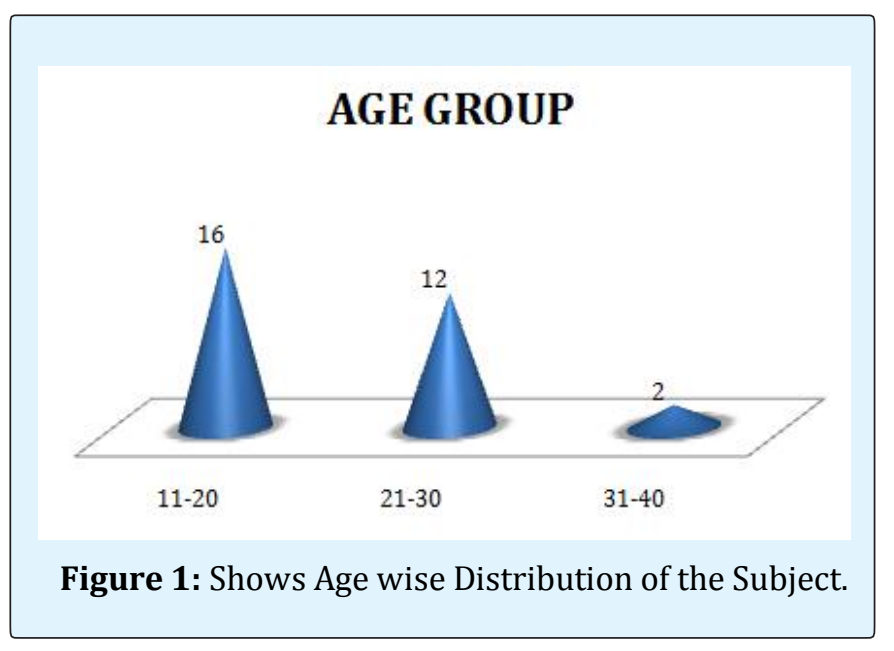

Partha Haradhan Chowdhury and Brinda Haren Shah. Effect of Visual Acuity on Ocular Deviation. J Ophthalmol 2018, 3(S1): 000S1-008.
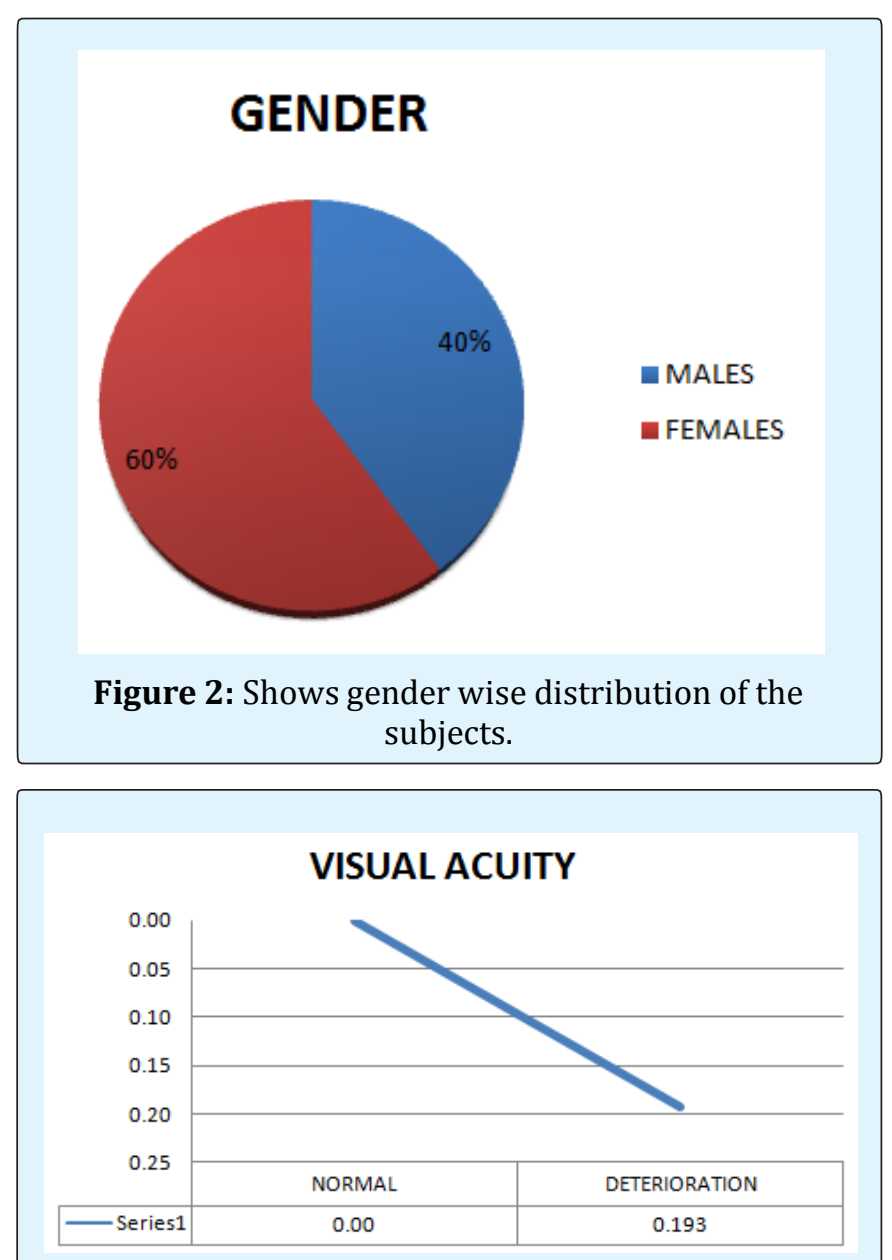

Figure 3: Shows comparison of mean visual acuity to the normal subject.

\section{Discussion}

Visual acuity of a normal individual is 0.00 log units. In cases of ocular deviation, visual acuity is reduced. As per the statistical analysis, mean visual acuity of 30 subjects is reduced to 0.193 log units in cases of ocular deviation. With increase in ocular deviation, visual acuity is decreased proportionately. It mainly occurs due to cone cell variation in macular region. The number of cone cells is highest in the foveal region compared to parafoveal region [5]. In cases of Eso deviation, deterioration is more as compared to Exo deviation $[6,7]$.

\section{Conclusion}

Visual acuity decreases significantly in cases of ocular deviation.

Copyright@ Partha Haradhan Chowdhury and Brinda Haren Shah. 


\section{Open Access Journal of Ophthalmology}

\section{References}

1. Anika K Tandon, Federico G Velez, Sherwin J Isenberg, Joseph L Demer, Stacy L Pineles (2014) Binocular Inhibition in Strabismic Patients is Associated with Diminished Quality of Life. Journal of American Association for Pediatric Ophthalmology and Strabismus 18(5): 423-426.

2. Kenneth $\mathrm{W}$ Wright, Peter H Spiegel, Lisa Thompson (2006) Handbook of Pediatric Strabismus and Amblyopia. $1^{\text {st }}$ (Edn.).

3. Zhale Rajavi, Sabbaghi H, Baghini AS, Yaseri M, Sheibani K, et al (2015) Prevalence of Colour Vision Deficiency and its Correlation with Amblyopia and Refractive Errors among Primary School Children. J Ophthalmic Vis Res 10(2): 130-138.
4. Hui Zhu, Jia-Jia Yu, Rong-Bin Yu, Hui Ding, Jing Bai, et al. (2015) Association between Childhood Strabismus and Refractive Error in Chinese Preschool Children. Plos One 10(3): e0120720.

5. Ye XC, Pegado V, Patel MS, Wasserman WW (2014) Strabismus genetics across a spectrum of eye misalignment disorders. Journal of clinical genetics 86(2): 103-111.

6. Kocak Altintas AG. (2000) Visual Acuity and Colour Vision deficiency in Amblyopia. European Journal of Ophthalmology 10(1): 77-81

7. Freeman AW, Nguyen VA, Jolly N (1996) Components of Visual Acuity Loss in Strabismus. Vision Research 36(5): 765-774. 